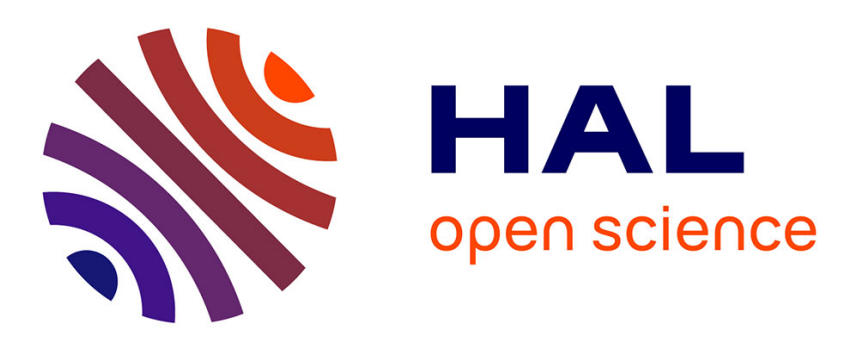

\title{
A note on the computation of the extrema of Young's modulus for hexagonal materials: an approach by planar tensor invariants
}

Paolo Vannucci

\section{- To cite this version:}

Paolo Vannucci. A note on the computation of the extrema of Young's modulus for hexagonal materials: an approach by planar tensor invariants. 2014. hal-01107516v2

\section{HAL Id: hal-01107516 \\ https://hal.science/hal-01107516v2}

Preprint submitted on 17 May 2015

HAL is a multi-disciplinary open access archive for the deposit and dissemination of scientific research documents, whether they are published or not. The documents may come from teaching and research institutions in France or abroad, or from public or private research centers.
L'archive ouverte pluridisciplinaire HAL, est destinée au dépôt et à la diffusion de documents scientifiques de niveau recherche, publiés ou non, émanant des établissements d'enseignement et de recherche français ou étrangers, des laboratoires publics ou privés. 


\title{
A note on the computation of the extrema of Young's modulus for hexagonal materials: an approach by planar tensor invariants
}

\author{
Paolo Vannucci \\ Université Paris-Saclay \\ LMV - Laboratoire de Mathématiques de Versailles, UMR 8100 \\ Université de Versailles Saint-Quentin en Yvelines \\ 45, Avenue des Etats-Unis. F-78035 Versailles, France. \\ paolo.vannucci@uvsq.fr
}

\begin{abstract}
A simple method to obtain the highest and lowest Young's modulus for a material of the hexagonal class is presented. It is based upon the use of tensor invariants of plane anisotropic elasticity; in fact, the cylindrical symmetry of the elastic tensor allows for transforming the 3D original problem into a planar one, with a considerable simplification.
\end{abstract}

Keywords: linear elasticity, anisotropy, polar formalism, bounds on elastic moduli

2010 MSC: 74B05, 74E10

\section{Introduction}

The problem of finding the extrema of the Young's modulus for materials belonging to the hexagonal class has been recently addressed by some authors, [1, 2, 3]. In particular, the last work by Cazzani clearly states some historical contributions going back to the XIX century, namely to the works of Saint Venant, $[4,5,6]$.

The purpose of this paper is to show that, thanks to the particular elastic symmetry of hexagonal materials, the problem of finding the extrema of the Young's modulus, as well as of any other elastic parameter, can be found by a direct bi-dimensional approach, much easier and straightforward than the general approaches proposed in the cited papers. Such an approach is based 
upon the polar formalism, where tensor invariants and angular variables are used to represent the elastic behavior of the material, in a way that reveals to be really powerful to state and solve the problem.

\section{Statement of the problem by the polar formalism}

Let us consider an elastic material having a hexagonal symmetry with $X_{3}$ as the axis of elastic symmetry. We address here the question of determining the extrema of the Young's modulus.

From a purely elastic point of view, a hexagonal symmetry cannot be distinguished from transverse isotropy: they share the same type of elastic tensors. This means also that for a hexagonal material, the elastic behavior is the same in all the meridian planes, i.e. those containing the $X_{3}$ axis.

For the purposes of this paper, this implies that it is sufficient to study the Young's modulus variation in any of such planes: the problem can be completely reduced from a three-dimensional to a planar one. For the sake of simplicity, we will indicate by $x$ and $y$ a couple of Cartesian axes in such a plane. Unlike what is commonly done in the literature, where the axis of symmetry for a material of the hexagonal symmetry class is labelled as the axis of $X_{3}$, the remaining two axes of $X_{1}$ and $X_{2}$ laying in the transversal plane, the use of axes labelled $x$ and $y$ has been preferred here, for emphasizing the planar character of the approach. These axes lay in a meridian plane; the axis of $x$ is rotated through an angle $\theta$ with respect to the transversal plane, hence, for instance, with respect to the $X_{1}$ or $X_{2}$ axes. For the sake of shortness, and for giving a particular importance to the couple of axes of orthotropy, we will name $x_{1}$ and $x_{2}$ the axes of $x$ and $y$, respectively, when $\theta=0$. Hence, for being more precise, $x_{1}=X_{1}$ (or, indifferently, $X_{2}$ ) and $x_{2}=X_{3}$, see Fig. 1. To remark that angle $\theta$ is here the latitude, i.e. $\theta=0$ corresponds to the equator and $\theta=\pi / 2$ to the pole.

It is worth to recall that the components of the compliance tensor $\mathbb{S}$ are exactly the same as those of the corresponding material in a planar state of stress. As $E(\theta)$ is given by (Voigt's notation is used throughout all this paper)

$$
E(\theta)=\frac{1}{S_{x x}(\theta)},
$$

the final question is hence reduced to a planar elastic one, depending on only one variable: to find the extrema of the Young's modulus $E(\theta)$ of a planar 


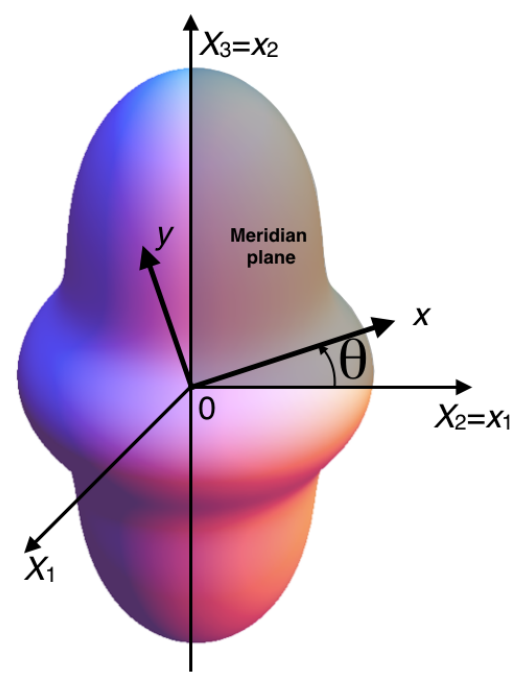

Figure 1: Sketch of the reference frames.

orthotropic material. For the mathematical symmetries, the study can be restricted to the set $0 \leq \theta<\pi / 2$.

An interesting tool for the study of planar problems in anisotropic elasticity is the polar formalism, introduced by Verchery in 1979, [7]. This method is particularly effective in several situations, namely because a tensor is described making use of invariants and angles only, which reveals to be very useful for describing anisotropic properties. Restricting here the attention to the strict topic of this paper (the interested reader can find a detailed account of the polar formalism in [8]; here, due to the peculiarity of the matter, only the case of orthotropic materials is considered), it is

$$
S_{x x}(\theta)=t_{0}+2 t_{1}+(-1)^{k} r_{0} \cos 4\left(\varphi_{1}-\theta\right)+4 r_{1} \cos 2\left(\varphi_{1}-\theta\right),
$$

with $t_{0}$ and $t_{1}$ the isotropy invariants, $r_{0}, r_{1}$ and $k$ the anisotropy invariants; all of them are non negative quantities. The last polar parameter, $\varphi_{1}$, is an angle that fixes the frame.

The expressions of the above quantities are given as functions of the Cartesian components of $\mathbb{S}$ by the following relations (with the usual frame $\left\{X_{1}, X_{2}, X_{3}\right\}$ mentioned above, $S_{22}$ should be $S_{33}, S_{12}$ should be $S_{13}$ or $S_{23}$ 
and $S_{66}$ indifferently $S_{44}$ or $S_{55}$, while $S_{11}$ does not change of indexes):

$$
\begin{gathered}
t_{0}=\frac{1}{8}\left(S_{11}-2 S_{12}+S_{66}+S_{22}\right), \\
t_{1}=\frac{1}{8}\left(S_{11}+2 S_{12}+S_{22}\right), \\
r_{0}=\frac{1}{8}\left|S_{11}-2 S_{12}-S_{66}+S_{22}\right|, \\
r_{1}=\frac{1}{8}\left|S_{11}-S_{22}\right| \\
\cos 2 \varphi_{1}=\frac{S_{11}-S_{22}}{\left|S_{11}-S_{22}\right|} .
\end{gathered}
$$

It can be proved that the fifth invariant, $k$, can only be an integer; thanks to the periodicity of the Cartesian components, see for instance eq. (2), only two values are actually interesting, 0 and 1 . For this reason, a suitable expression for uniquely determining $k$ is:

$$
k=\frac{1}{2}\left|\frac{S_{11}-2 S_{12}-S_{66}+S_{22}}{\left|S_{11}-2 S_{12}-S_{66}+S_{22}\right|}-1\right| ;
$$

$k$ fixes the type of planar orthotropy and is of the greatest importance in the following, see also [9].

Incidentally, a glance at eq. (2) shows that the Cartesian components of $\mathbb{S}$ needed to describe the behavior of the material in a meridian plane are four, but that actually this behavior depends on all the five tensor invariants of the fictitious planar orthotropic material having the same Cartesian components of the hexagonal-symmetric material (i.e., with $S_{22}$ corresponding to $S_{33}, S_{66}$ to $S_{44}$ and so on, as explained above). So, the planar analysis depends on the whole set of independent invariants and in this sense it is hence complete.

A remark, for the sake of precision: formulae in eq. (3) and (4) have been written as functions of the Cartesian components in the frame $x_{1}-x_{2}$, i.e. for $\theta=0$, and considering an orthotropic material, which is the case of concern here. More general formulae, using the Cartesian components in a generic frame $x-y$ rotated through an angle $\theta$ and concerning a completely anisotropic material, could be written as well, but have been omitted here for the sake of conciseness.

Of course, see eq. (1), the extrema of $S_{x x}(\theta)$ correspond to those of $E(\theta)$ : a maximum for $S_{x x}(\theta)$ is a minimum for $E(\theta)$, and a minimum for $S_{x x}(\theta)$ is a maximum for $E(\theta)$. Hence, we will study $S_{x x}(\theta)$, the results for $E(\theta)$ will descend immediately.

Before proceeding with the search for the extrema of $S_{x x}(\theta)$, let us turn the attention on the choice of the frame. Fixing $\varphi_{1}=0$ corresponds to 
put the axis of $x_{1}$ aligned with the direction giving the highest value of $S_{x x}(\theta), \theta \in\{0, \pi\}$, and $\varphi_{1}=\pi / 2$ with the direction of the lowest value. Because these are the only two cases of concern here, it is worth to write eq. (2) in the equivalent form

$$
S_{x x}(\theta)=t_{0}+2 t_{1}+(-1)^{k} r_{0} \cos 4 \theta+4(-1)^{h} r_{1} \cos 2 \theta,
$$

with $h=0$ if $\varphi_{1}=0$ and $h=1$ if $\varphi_{1}=\pi / 2$. A suitable Cartesian expression for $h$ is

$$
h=\frac{1}{2}\left|\frac{S_{11}-S_{22}}{\left|S_{11}-S_{22}\right|}-1\right| .
$$

Looking at eq. $\left(3_{5}\right)$, it can be easily noticed that for materials with $S_{11}>S_{22}, \varphi_{1}=0$ and $h=0$. This is the case of a Young's modulus represented by a prolate surface, i.e. having $E(\theta=0)<E(\theta=\pi / 2)$; to remember that the $x_{1}$ axis belongs to the transversal plane and $x_{2}$ is the hexagonal axis of symmetry of the material. An oblate surface, i.e. with $E(\theta=0)>E(\theta=\pi / 2)$, corresponds to the case $S_{11}<S_{22}$, that gives $\varphi_{1}=\pi / 2$ and $h=1$.

Though parameter $h$ plays an algebraic role equivalent to that of $k$, nonetheless, these two quantities are really different, mathematically and mechanically: $k$ is an invariant that determines the type of orthotropy regardless of the frame, $h$ is a parameter linked to the frame choice that gives the form of the surface of $E$ : prolate, for $h=0$, oblate for $h=1$.

The first derivative of $S_{x x}(\theta)$ is

$$
S_{x x}^{\prime}(\theta)=-8 r_{1}\left[(-1)^{k} \rho \cos 2 \theta+(-1)^{h}\right] \sin 2 \theta,
$$

where

$$
\rho=\frac{r_{0}}{r_{1}}
$$

The zeros of $S_{x x}^{\prime}(\theta)$ are

$$
\theta_{1}=0, \quad \theta_{2}=\frac{1}{2} \arccos \frac{(-1)^{1+h-k}}{\rho}, \quad \theta_{3}=\frac{\pi}{2}
$$

the second (intermediate) root, $\theta_{2}$, exists $\Longleftrightarrow \rho>1$. The second derivative of $S_{x x}(\theta)$ is

$$
S_{x x}^{\prime \prime}(\theta)=-16 r_{1}\left[(-1)^{k} \rho \cos 4 \theta+(-1)^{h} \cos 2 \theta\right],
$$


that gets the following values for the zeros of $S_{x x}^{\prime}(\theta)$ :

$$
\begin{gathered}
S_{x x}^{\prime \prime}\left(\theta_{1}\right)=-16 r_{1}\left[(-1)^{k} \rho+(-1)^{h}\right], \\
S_{x x}^{\prime \prime}\left(\theta_{2}\right)=-16(-1)^{k} r_{1} \frac{1-\rho^{2}}{\rho}, \\
S_{x x}^{\prime \prime}\left(\theta_{3}\right)=-16 r_{1}\left[(-1)^{k} \rho-(-1)^{h}\right] .
\end{gathered}
$$

When $\theta_{2}$ exists, $\rho>1$, so it is always

$$
\frac{1-\rho^{2}}{\rho}<0
$$

hence, the sign of $S_{x x}^{\prime \prime}\left(\theta_{2}\right)$ depends exclusively on $k$, because $r_{1} \geq 0$, unlike what happens for $S_{x x}^{\prime \prime}\left(\theta_{1}\right)$ and $S_{x x}^{\prime \prime}\left(\theta_{3}\right)$, where it depends also on $h$, hence on the form of the surface representing $E$.

Considering the above equations and remarks, one can easily determine the nature of the stationary points of $S_{x x}(\theta)$ and hence of $E(\theta)$ :

$$
\begin{aligned}
& E\left(\theta_{1}\right)=\frac{1}{t_{0}+2 t_{1}+(-1)^{k} r_{0}+4(-1)^{h} r_{1}}, \\
& E\left(\theta_{2}\right)=\frac{1}{t_{0}+2 t_{1}-(-1)^{k}\left(r_{0}^{2}+2 r_{1}^{2}\right) / r_{0}}, \\
& E\left(\theta_{3}\right)=\frac{1}{t_{0}+2 t_{1}+(-1)^{k} r_{0}-4(-1)^{h} r_{1}} .
\end{aligned}
$$

The results for all the possible cases, eight on the whole, are summarized in Table 1, along with eight examples of materials, extracted from [3]; Be is Beryllium, Tc is Technetium, Ti is Titanium, CdS is Cadmium-Sulphide, $\mathrm{Cd}-\mathrm{Zn}$ is a Cadmium-Zinc alloy, $\mathrm{Zn}$ is Zinc, InSe is Indium Selenide and $\mathrm{Cd}-\mathrm{Mg}$ is a Cadmium-Magnesium alloy. It is worth noticing that the same extremal value of $E(\theta)$ can change its nature as a function of $\rho$, while $E\left(\theta_{2}\right)$ does not depend upon $h$, confirming the remark above.

The expressions of $E\left(\theta_{i}\right)$ in eq. (13) can be given in a completely Cartesian form using eq. (3):

$$
\begin{gathered}
E\left(\theta_{1}\right)=\frac{1}{S_{11}}, \\
E\left(\theta_{2}\right)=\frac{4\left(S_{11}-2 S_{12}-S_{66}+S_{22}\right)}{4 S_{11} S_{22}-\left(2 S_{12}+S_{66}\right)^{2}}, \\
E\left(\theta_{3}\right)=\frac{1}{S_{22}} .
\end{gathered}
$$

It should be remarked that the difference between the cases $k=0$ and $k=1$ does not appear explicitly with the Cartesian formalism. Anyway, eq. (4), it is easy to check that

$$
k=0 \Longleftrightarrow S_{11}-2 S_{12}-S_{66}+S_{22}>0 .
$$


Table 1: Extrema of the Young's modulus: polar formulae.

\begin{tabular}{|c|c|c|c|c|c|}
\hline & & $\theta_{1}$ & $\theta_{2}$ & $\theta_{3}$ & Example \\
\hline \multirow{10}{*}{$h=0$} & \multicolumn{5}{|c|}{$k=0$} \\
\hline & $\rho \leq 1$ & $\min$ & - & $\max$ & $\mathrm{Be}$ \\
\hline & $\rho>1$ & absolute min & $\max$ & local min & Tc \\
\hline & & & $\left(\theta_{2} \in\{\pi / 4, \pi / 2\}\right)$ & & \\
\hline & $E\left(\theta_{i}\right)$ & $\frac{1}{t_{0}+2 t_{1}+r_{0}+4 r_{1}}$ & $\frac{1}{t_{0}+2 t_{1}-r_{0}-2 r_{1}^{2} / r_{0}}$ & $\frac{1}{t_{0}+2 t_{1}+r_{0}-4 r_{1}}$ & \\
\hline & \multicolumn{5}{|c|}{$k=1$} \\
\hline & $\rho \leq 1$ & $\min$ & - & $\max$ & $\mathrm{Ti}$ \\
\hline & $\rho>1$ & local max & $\min$ & absolute max & $\mathrm{CdS}$ \\
\hline & & & $\left(\theta_{2} \in\{0, \pi / 4\}\right)$ & & \\
\hline & $E\left(\theta_{i}\right)$ & $\frac{1}{t_{0}+2 t_{1}-r_{0}+4 r_{1}}$ & $\frac{1}{t_{0}+2 t_{1}+r_{0}+2 r_{1}^{2} / r_{0}}$ & $\frac{1}{t_{0}+2 t_{1}-r_{0}-4 r_{1}}$ & \\
\hline \multirow{10}{*}{$h=1$} & \multicolumn{5}{|c|}{$k=0$} \\
\hline & $\rho \leq 1$ & $\max$ & - & $\min$ & $\mathrm{Cd}-\mathrm{Zn}$ \\
\hline & $\rho>1$ & local min & $\max$ & absolute min & $\mathrm{Zn}$ \\
\hline & & & $\left(\theta_{2} \in\{0, \pi / 4\}\right)$ & & \\
\hline & $E\left(\theta_{i}\right)$ & $\frac{1}{t_{0}+2 t_{1}+r_{0}-4 r_{1}}$ & $\frac{1}{t_{0}+2 t_{1}-r_{0}-2 r_{1}^{2} / r_{0}}$ & $\frac{1}{t_{0}+2 t_{1}+r_{0}+4 r_{1}}$ & \\
\hline & \multicolumn{5}{|c|}{$k=1$} \\
\hline & $\rho \leq 1$ & $\max$ & - & $\min$ & InSe \\
\hline & $\rho>1$ & absolute max & $\min$ & local max & $\mathrm{Cd}-\mathrm{Mg}$ \\
\hline & & & $\left(\theta_{2} \in\{\pi / 4, \pi / 2\}\right)$ & & \\
\hline & $E\left(\theta_{i}\right)$ & $\frac{1}{t_{0}+2 t_{1}-r_{0}-4 r_{1}}$ & $\frac{1}{t_{0}+2 t_{1}+r_{0}+2 r_{1}^{2} / r_{0}}$ & $\frac{1}{t_{0}+2 t_{1}-r_{0}+4 r_{1}}$ & \\
\hline
\end{tabular}


About the angle $\theta_{2}$, its value is

$$
\theta_{2}=\frac{1}{2} \arccos \frac{(-1)^{1+h-k}}{\rho}=\frac{1}{2} \arccos \frac{S_{22}-S_{11}}{S_{11}-2 S_{12}-S_{66}+S_{22}} .
$$

Its polar expression gives immediately that for $k \neq h, 0 \leq \theta_{2}<\pi / 4$, while for $k=h, \pi / 4 \leq \theta_{2}<\pi / 2$. Hence, the intermediate minimum of $E(\theta)$ is located between 0 and $\pi / 4$, while the intermediate maximum between $\pi / 4$ and $\pi / 2$ for $h=0$, prolate surfaces, and the exact contrary for the oblate surfaces, $h=1$, see Table 1 .

It is evident that angle $\theta_{2}$, and hence the second (intermediate) stationary value of the Young's modulus, exists if and only if the argument of function arccos in eq. (16) is admissible, i.e. if and only if

$$
\rho>1 \Longleftrightarrow r_{0}>r_{1}
$$

or equivalently if and only if

$$
\left|S_{22}-S_{11}\right|<\left|S_{11}-2 S_{12}-S_{66}+S_{22}\right| .
$$

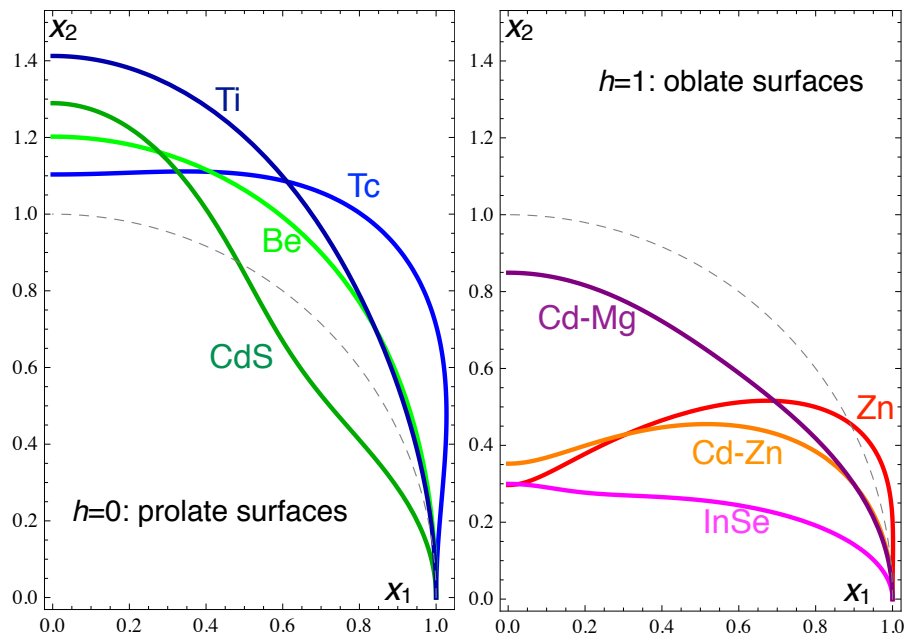

Figure 2: Normalized directional diagrams of $E(\theta)$ for the eight materials in Table 2. The dashed line represents the isotropic case

Eight examples, referring to the materials presented in Table 1, are presented in Table 2. The variation of the $E(\theta)$ for these eight cases is presented 
Table 2: Numerical values for the eight materials cited in Table 1; the Young's moduli are in $\mathrm{GPa}$, the Cartesian and polar components of $\mathbb{S}$ in $\mathrm{GPa}^{-1} \times 10^{-3}$.

\begin{tabular}{lrrrrrrrr}
\hline & $\mathbf{B e}$ & $\mathbf{T c}$ & $\mathbf{T i}$ & $\mathbf{C d S}$ & $\mathbf{C d}-\mathbf{Z n}$ & $\mathbf{Z n}$ & $\mathbf{I n S e}$ & $\mathbf{C d}-\mathbf{M g}$ \\
\hline$S_{11}$ & 3.45 & 3.20 & 9.69 & 20.50 & 12.55 & 8.22 & 11.60 & 24.20 \\
$S_{22}$ & 2.87 & 2.90 & 6.86 & 15.90 & 35.62 & 27.70 & 38.70 & 28.50 \\
$S_{12}$ & -0.05 & -0.90 & -1.82 & -5.30 & -9.76 & -7.00 & -7.50 & -6.20 \\
$S_{66}$ & 6.16 & 5.70 & 21.50 & 66.70 & 49.78 & 25.30 & 85.50 & 75.10 \\
\hline$t_{0}$ & 1.57 & 1.70 & 5.21 & 14.21 & 14.68 & 9.40 & 18.85 & 17.53 \\
$t_{1}$ & 0.78 & 0.54 & 1.61 & 3.23 & 3.58 & 2.74 & 4.41 & 5.04 \\
$r_{0}$ & 0.03 & 0.28 & 0.16 & 2.46 & 2.24 & 3.08 & 2.53 & 1.25 \\
$r_{1}$ & 0.07 & 0.04 & 0.35 & 0.58 & 2.88 & 2.44 & 3.39 & 0.54 \\
$k$ & 0 & 0 & 1 & 1 & 0 & 0 & 1 & 1 \\
$h$ & 0 & 0 & 0 & 0 & 1 & 1 & 1 & 1 \\
$\rho$ & 0.45 & 7.33 & 0.46 & 4.28 & 0.78 & 1.26 & 0.75 & 2.33 \\
\hline$\theta_{2}\left(^{\circ}\right)$ & - & 48.92 & - & 38.25 & - & 18.85 & - & 57.73 \\
$E\left(\theta_{1}\right)$ & 289.86 & 312.50 & 103.20 & 48.78 & 79.68 & 121.65 & 86.21 & 41.32 \\
$E\left(\theta_{2}\right)$ & - & 401.64 & - & 42.75 & - & 125.76 & - & 34.12 \\
$E\left(\theta_{3}\right)$ & 348.43 & 344.83 & 145.77 & 62.89 & 28.07 & 36.10 & 25.84 & 35.09 \\
\hline
\end{tabular}

in Fig. 2; the diagrams have been normalized with respect to $E(\theta=0)$. In Fig. 3 the three-dimensional surface diagram of $E$ is presented for these eight materials; following a consolidated habitude, the symmetry axis has been put in the vertical direction.

\section{Conclusion}

The problem of determining the extrema of the Young's modulus has been addressed in this paper; it has been shown that it can be reduced to the search for the maxima and minima of the component $S_{x x}$ of the compliance tensor $\mathbb{S}$ of an ordinarily orthotropic planar material. In such a case, the use of the polar tensor invariants reduces the problem to an almost trivial one, with the advantage that the different possible cases, actually only eight, are determined by three non negative dimensionless parameters: $k$, an inte- 

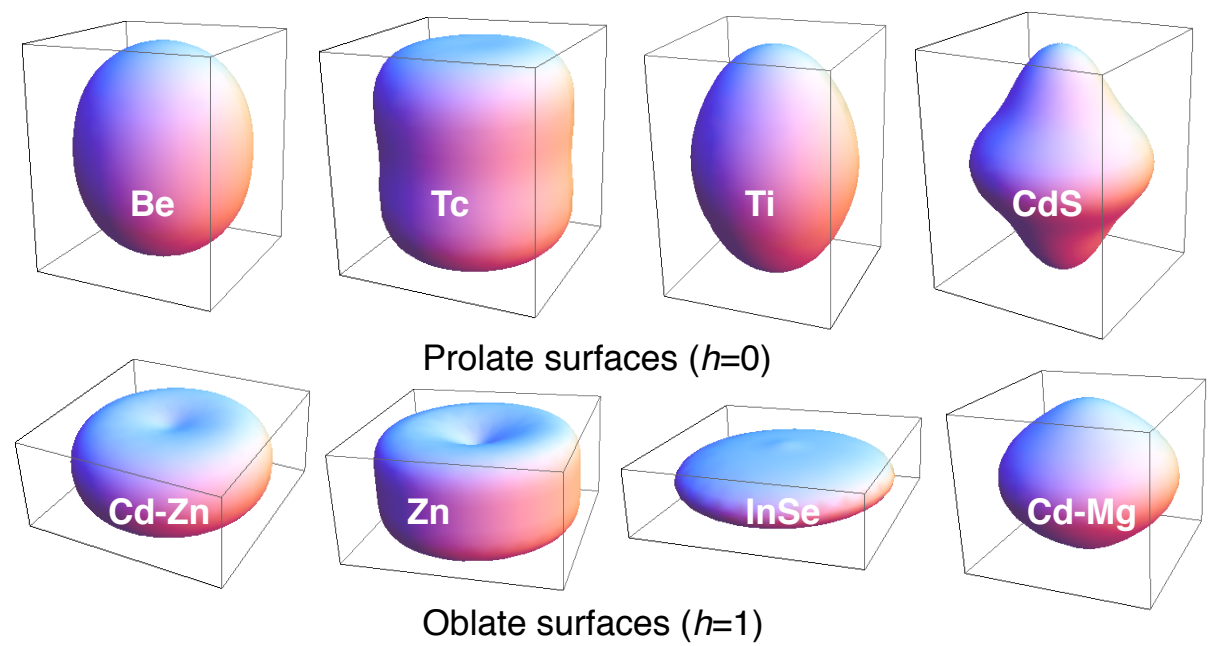

Figure 3: Normalized three-dimensional diagrams of $E$ for the eight materials in Table 2.

ger invariant accounting for the type of planar ordinary orthotropy, $h$, still an integer, taking into account for the type of the surface representing $E$, and the ratio $\rho$, representing the relative importance of the two compliance anisotropy phases, $r_{0}$ and $r_{1}$.

\section{References}

[1] A. Cazzani, M. Rovati, Extrema of Young's modulus for cubic and transversely isotropic solids, International Journal of Solids and Structures 40 (2003) 1713-1744.

[2] R. A. Khan, F. Ahmad, Extrema of Young's modulus in hexagonal materials, Applied Mathematics and Computation 219 (2012) 2260-2266.

[3] A. Cazzani, On the true extrema of Young's modulus in hexagonal materials, Applied Mathematics and Computation 238 (2014) 397-407.

[4] A.-J.-C. Barré de Saint Venant, Mémoire sur la distribution des élasticités autour de chaque point d'un solide ou d'un milieu de contexture quelconque, particulièrement lorsqu'il est amorphe sans être 
isotrope (Premier article), Journal de mathématiques pures et appliquées (Liouville) 8 series II (1863) 257-295.

[5] A.-J.-C. Barré de Saint Venant, Mémoire sur la distribution des élasticités autour de chaque point d'un solide ou d'un milieu de contexture quelconque, particulièrement lorsqu'il est amorphe sans être isotrope (Deuxième article), Journal de mathématiques pures et appliquées (Liouville) 8 series II (1863) 353-430.

[6] A.-J.-C. Barré de Saint Venant, Mémoire sur les divers genres d'homogénéité des corps solides, et principalement sur l'homogénéité semi-polaire ou cylindrique, et sur les homogénéités polaires ou sphériconique et sphérique, Journal de mathématiques pures et appliquées (Liouville) 10 series II (1867) 297-349.

[7] G. Verchery, Les invariants des tenseurs d'ordre 4 du type de l'élasticité. Proc. of Colloque Euromech 115, Villard-de-Lans. In Comportement mécanique des matériaux anisotropes, Editions du CNRS, Paris, (1982) 93-104.

[8] P. Vannucci, Plane anisotropy by the polar method. Meccanica 40 (2005) $437-454$.

[9] P. Vannucci, Influence of invariant material parameters on the flexural optimal design of thin anisotropic laminates. International Journal of Mechanical Sciences 51 (2009) 192-203. 